\title{
Water-Density Effects on Phenol Oxidation in Supercritical Water
}

\author{
Jeffrey T. Henrikson and Phillip E. Savage \\ Chemical Engineering Dept., University of Michigan, Ann Arbor, MI 48109
}

\begin{abstract}
Supercritical water oxidation ( $S C W O$ ) experiments were performed in a tubular flow reactor at $420-465^{\circ} \mathrm{C}$ and 141-241 bar. Phenol was the organic reactant. Both pure water and a helium (1/3 by mol)-water mixture served as reaction media. Adding helium to the reaction medium permitted variation of the water concentration and system pressure independently. By decoupling the water concentration from the system pressure, it was shown that the rate of phenol disappearance during SCWO is influenced by the water concentration and not the system pressure. The experiments consistently revealed that adding helium, and thereby decreasing the water concentration at a fixed system pressure, increased the phenol conversion. In addition, experiments showed that lowering the water concentration using pure water as the solvent also increased the phenol conversion. The results suggest that dilution with helium (and perhaps other inert gases) may be a new way to control SCWO reaction rates.
\end{abstract}

\section{Introduction}

Supercritical water oxidation (SCWO) is the complete oxidation of organic compounds in an aqueous phase at temperatures and pressures above the critical values for water $\left(374.15^{\circ} \mathrm{C}\right.$ and $\left.220.9 \mathrm{bar}\right)$. SCWO of hazardous organic materials to water and carbon dioxide was conceived of over two decades ago. The technology usually competes with incineration and wet-air oxidation. SCWO has advantages over wet-air oxidation, because of the single supercritical phase, shorter residence times (100-200 s), and more complete oxidation of difficult to oxidize compounds. SCWO is preferred over incineration for waste streams with high water content, due to the lower operating temperatures of SCWO $\left(380-650^{\circ} \mathrm{C}\right)$ and the favorable formation of molecular nitrogen rather than $\mathrm{NO}_{x}$.

SCWO chemistry has received attention from the kinetics community due to the potential advantages this technology offers. Several research groups have studied the process variables that influence the rate of SCWO [temperature, pressure, identity, and amount of the organic compound(s), catalyst(s), and oxidant]. The effects of these process variables have been elucidated, and they have the greatest im-

Correspondence concerning this article should be addressed to P. E. Savage. pact on the kinetics of a SCWO process. The research to date has not, however, explained fully the effect and role of water in the oxidation process. More than two decades after the birth of supercritical water oxidation, it remains unclear how and whether the water concentration increases or decreases the rate of organic-compound disappearance and subsequent oxidation to water and carbon dioxide. The ambiguity and apparent conflict in the literature is perhaps exemplified best by the situation for phenol SCWO. Several groups have noted that isothermal changes in the water concentration (or system pressure) influence the SCWO kinetics for phenol disappearance. In most reports, the reaction rate increased with the water density (Gopalan and Savage, 1995; Koo et al., 1997; Thornton and Savage, 1992). In one report, the oxidation rate decreased with increasing water density (Oshima et al., 1998). In yet another report, the oxidation rate was assumed to be independent of the water density (Krajnc and Levec, 1996).

If the question of the effect of water concentration on SCWO rates is expanded to include other compounds, then the apparent conflict grows, for water has been reported to increase (Holgate and Tester, 1994; Li et al., 1993; Martino and Savage, 1997; Martino et al., 1995; Meyer et al., 1995; 
Rice et al., 1998; Savage and Smith, 1995), decrease (Dell' Orco et al., 1995; Steeper et al., 1996), or have no effect on (Chang et al., 1993; Krajnc and Levec, 1997; Lee and Gloyna, 1992; Lee et al., 1997; Lee and Park, 1996) the oxidation rates of different organic compounds under supercritical conditions. There does not appear to be any common links between the reaction conditions used and the observed effect of water concentration on the rate.

In all but one of the previous phenol SCWO studies, the exception being Koo et al. (1997), the water density and system pressure were varied together. This was the case because these SCWO experiments used small amounts of organic reactant and small amounts of oxidant, so that over $99 \%$ of the molar mass was water. As a consequence, as the system pressure increased, the water density also increased. What makes the work of Koo et al. (1997) and that reported in this article different is that an inert gas was introduced into the reaction medium. By introducing helium into the reaction medium, the water concentration could be held constant, while the system pressure increased. Similarly, the water concentration could be reduced by the addition of helium at a fixed system pressure. Koo et al. (1997) performed batch experiments where the reactants were brought separately to the reaction temperature in a water or helium-water medium, and then mixed together in one reactor at a set pressure. The authors' conclusion was that at the conditions tested, it was the water density that affected the phenol disappearance and not the system pressure. Furthermore, they found that as the water density increased, the phenol disappearance rate also increased.

The present article comprises the largest data set to date in which the water concentration and system pressure were varied independently, and it represents the only work of this type performed in a tubular flow reactor. Moreover, this article provides results from a different and broader range of experimental conditions than those utilized by Koo et al. (1997). We have been able to isolate the separate roles of pressure and water concentration on the SCWO reaction rate for phenol.

\section{Experimental Studies}

We performed phenol SCWO experiments at three different temperatures $\left(420,442\right.$ and $\left.465^{\circ} \mathrm{C}\right)$, three different pressures (141, 207 and 241 bar), and in two different reaction media (pure water, and $1 / 3$ helium-2/3 water by mol). For

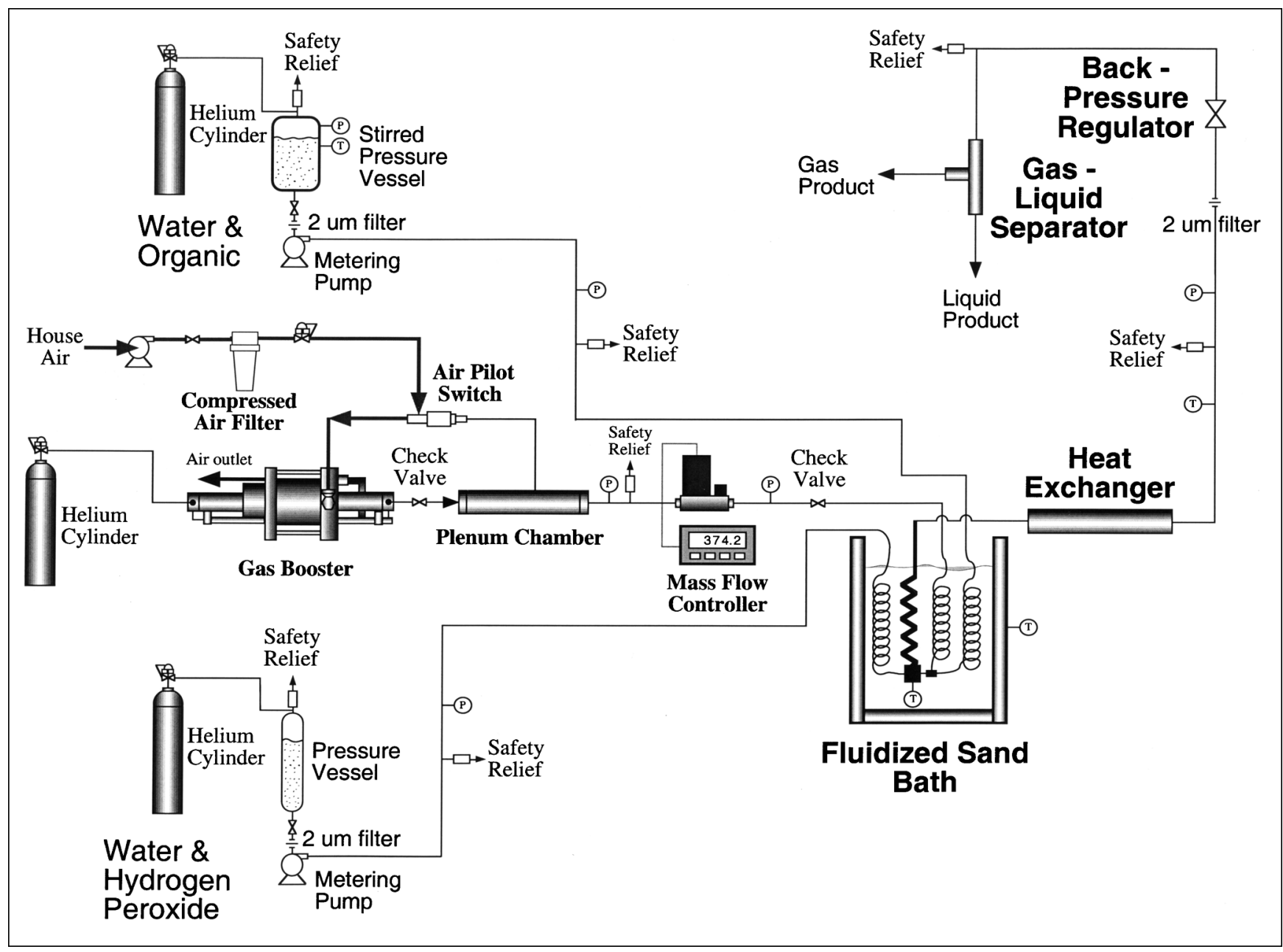

Figure 1. Experimental apparatus. 
each of the 18 temperature, pressure, medium combinations, we measured the phenol conversion and in some cases the $\mathrm{CO}$ and $\mathrm{CO}_{2}$ yields at different residence times in a tubular flow reactor. Figure 1 shows the experimental apparatus that was used to conduct the SCWO experiments. Nearly all experiments utilized the same initial phenol and oxygen concentrations of $[\phi \mathrm{OH}]_{o}=0.18 \pm 0.006 \mathrm{mmol} / \mathrm{L}$ and $\left[\mathrm{O}_{2}\right]_{o}=$ $6.7 \pm 0.19 \mathrm{mmol} / \mathrm{L}$, measured at reaction conditions. The uncertainties reported here and elsewhere in this article are at the $95 \%$ confidence level. The water and helium concentrations varied from $1.6 \mathrm{M}$ to $6.8 \mathrm{M}$ and $0.70 \mathrm{M}$ to $3.0 \mathrm{M}$, respectively.

The water used in these experiments was distilled, deionized, and deaerated prior to use. All chemicals and gases were obtained commercially and used as received. The nominal purities for phenol and helium were $99 \%$ and $99.995 \%$, respectively.

A known amount of phenol was mixed with a known amount of water and placed in a Parr model 4551 4-L stirred pressure vessel under 35 bar of helium pressure. This solution served as the phenol feed stream to the reactor. A known amount of a $30 \mathrm{wt} \% \mathrm{H}_{2} \mathrm{O}_{2}$ solution was mixed with a known amount of water and placed in a Whitey one-gallon highpressure cylinder under 35 bar of helium pressure. This solution served as the oxidant feed stream. The $\mathrm{H}_{2} \mathrm{O}_{2}$ decomposes completely to molecular oxygen and water as this stream is preheated to the reaction temperature. Phenix et al. (2002) demonstrated that experimental results are not affected by the use of $\mathrm{H}_{2} \mathrm{O}_{2}$ (rather than gaseous $\mathrm{O}_{2}$ ) as the source of oxygen.

Each aqueous solution was pumped through a separate 3.2 $\mathrm{m} \times 0.108 \mathrm{~cm}$ ID Hastelloy preheater line to the reactor using separate Eldex model AA-100-S metering pumps with a nominal flow-rate range of $1.1-9.8 \mathrm{~mL} / \mathrm{min}$. In some experiments, helium was fed to the reactor in a separate $3.8 \mathrm{~m} \times$ $0.108 \mathrm{~cm}$ ID preheater line using a model AGT-30/75, two stage, Haskel gas booster to obtain the desired pressure, and a model 5850TR flowmeter and a model 0151E mass flow controller from Brooks Instruments to regulate the amount of helium entering the preheater line. The nominal mass flow-rate range of the flowmeter is $0.02-2.0 \mathrm{~g}$ of helium per minute. The helium flow rate was verified by experimental measurement at the reactor exit. We used the guidelines set forth by Marrone et al. (1998) to determine the length of the preheater lines required for adequate preheating of the feed streams, and verified their adequacy experimentally by measuring the fluid temperature at the reactor entrance.

The oxidant and helium preheater lines meet in a stainless-steel, three-way mixing tee from HiP (High Pressure Equipment Company). The oxidant/helium mixture then passes to a Hastelloy, four-way mixing tee from HiP. At this point the oxidant/helium stream mixes with the phenol-containing stream. The last two ports in the four-way mixing tee provide access to the tubular reactor and a thermocouple that measures the fluid temperature at the reactor entrance.

In experiments without helium, the reactant streams were always within $\pm 1 \mathrm{~K}$ of the nominal reaction temperature as they mixed and entered the tubular reactor. In the experiments with added helium, the reactant streams were also within $\pm 1 \mathrm{~K}$ of the nominal reaction temperature at low flow rates, but as the flow rate increased, the temperature difference increased to as much as $6 \mathrm{~K}$. The larger temperature difference with added helium is due to the large flow rate of helium through the tubing. Extending the helium preheater line from $3.8 \mathrm{~m}$ to $5 \mathrm{~m}$ did not eliminate this problem. Therefore, some of the experiments performed with helium at short residence times were likely done at a temperature slightly below the nominal reaction temperature. This modest temperature difference does not affect any of the conclusions we will draw from the experimental data, because lower temperatures would suppress phenol conversion, whereas we observe that adding helium increased phenol conversion in all instances.

The tubular reactor is composed of Hastelloy and has a volume of $68 \mathrm{~cm}^{3}$, with two separate tubing lengths and internal diameters. The first section of the reactor $(91 \%$ of the total volume) has a length of $11.0 \mathrm{~m}$ and an ID of $0.27 \mathrm{~cm}$, while the last section of the reactor has a length of $4.1 \mathrm{~m}$ and an ID of $0.14 \mathrm{~cm}$. Two tubing lengths were used for convenience. We fabricated this reactor by adding the 11-m tube to an existing reactor. A longer reactor was necessary to obtain residence times that spanned a large range of phenol conversions for both water and helium-water reaction media. The volume of this reactor ranged from being 1.7 to 10 times larger than any previous reactor used to study phenol SCWO (Gopalan and Savage, 1995; Koo et al., 1997; Thornton and Savage, 1992; Oshima et al., 1998; Krajnc and Levec, 1996). The preheater lines and the reactor are heated in a temperature-controlled, isothermal Techne SBL-2D fluidized sand bath.

After leaving the reactor, the reaction mixture is quickly quenched using a cocurrent tube-in-tube heat exchanger with cooling water in the annulus. The product stream then passes through a Tescom model 54 back-pressure regulator and is returned to ambient pressure. The liquid and gas phases separate at ambient conditions in a glass tee, and the flow rates of both phases are measured.

The gas phase is analyzed on-line for oxygen, carbon monoxide, and carbon dioxide using a gas chromatograph (GC). The GC is equipped with a thermal conductivity detector (TCD) and a 15-ft Supelco Carboxen 1,000 column that is packed with $60 / 80$ mesh particles. Helium serves as both the carrier gas and as the reference gas for the analysis. A known volume of the gas sample is injected into the column via a Valco valve. The initial oven temperature of $35^{\circ} \mathrm{C}$ is held for $5 \mathrm{~min}$ and then increased by $20^{\circ} \mathrm{C} / \mathrm{min}$ up to $225^{\circ} \mathrm{C}$. The final temperature is then maintained for $6 \mathrm{~min}$.

The liquid effluent is collected for subsequent analysis by a high-performance liquid chromatograph (HPLC) to determine the final phenol concentrations. The HPLC analyzes a $10-\mu \mathrm{L}$ sample. Sample constituents are separated on a Supelco LC-18 column with an internal diameter of $4.6 \mathrm{~mm}$, a length of $25 \mathrm{~cm}$, and a particle size of $5 \mu \mathrm{m}$, and detected by an ultraviolet detector that operates at $210 \mathrm{~nm}$. The mobile phase of $72 \%$ water and $28 \%$ acetonitrile by volume flows at $1 \mathrm{~mL} / \mathrm{min}$ at a pressure of 2,000 psi. A typical analysis requires $10 \mathrm{~min}$, and the phenol peak typically occurs around 7 $\min$.

\section{Phase Behavior and Fluid Properties}

When conducting chemical reactions in supercritical fluids, it is good practice to examine the phase behavior of the flu- 
ids. The fluid phase in the oxidation experiments without helium was always at least $99+\%$ water, so we assumed that the critical temperature of the mixture was close to the critical temperature of pure water $\left(374^{\circ} \mathrm{C}\right)$. Thus, all of the experiments without helium were done in the supercritical (singlephase) region. Additionally, we took the density of the mixture to be the density of water at the same conditions (Haar et al., 1984).

The situation was more complicated for the experiments in helium-water mixtures. Sretenskaja et al. (1995) provide experimental data for the critical points of such mixtures up to $35 \%$ helium. The critical temperature for a $33.3 \%$ helium mixture, which we used in the experiments, was interpolated to be $417^{\circ} \mathrm{C}$. The lowest temperature we used in the experiments is $420^{\circ} \mathrm{C}$, so all experiments with helium-water mixtures were done above the mixture critical temperature. Thus, these experiments were also done in a single supercritical phase. As a check, the critical temperature of a $65 \%$ water-35\%-helium mixture was calculated using the method of Stradi et al. (2001) with the Peng-Robinson (PR) and Soave-Redlich-Kwong (SRK) cubic equations of state. The critical temperature was calculated to be $413^{\circ} \mathrm{C}(\mathrm{PR})$ and $405^{\circ} \mathrm{C}$ (SRK). These estimates are both lower than the experimental value of $423^{\circ} \mathrm{C}$ (Sretenskaja et al., 1995), and most likely reflect the inability of the cubic equations of state to model adequately a polar, associating fluid like water. Sretenskaja et al. (1995) also measured the density of helium-water mixtures from $410-450^{\circ} \mathrm{C}, 60-200 \mathrm{MPa}$, and $0-100 \%$ helium, and compared the experimental mixture densities with densities calculated from the Guggenheim, Heilig-Franck, and van der Waals equations of state. Surprisingly, the van der Waals equation of state represented the data the most accurately, so we used it to calculate, by extrapolation, the density of the helium-water mixtures in our experiments. The density, in turn, was used along with the reactor volume, and measured flow rates to determine the residence time for each experiment.

\section{Results and Discussion}

This section successively discusses different independent variables and the effect each had on the phenol conversion during the SCWO experiments. The primary goal was to gain insight into the role that the water concentration and system pressure have on the rate of phenol oxidation. It is not our

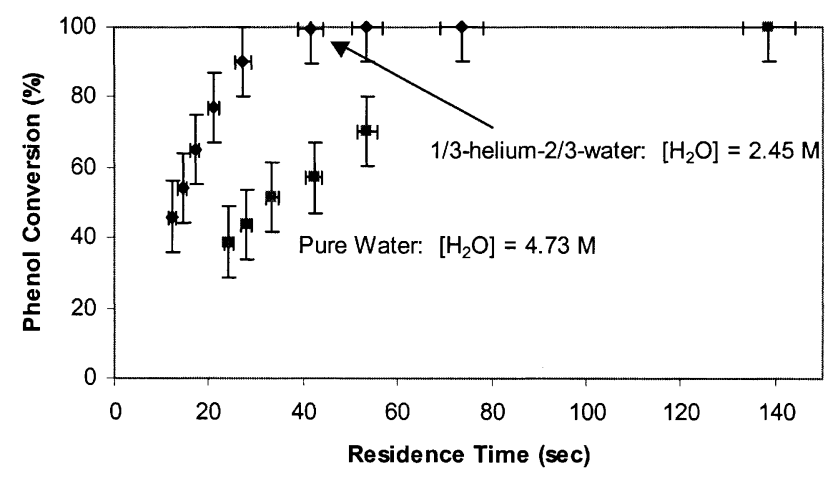

Figure 2. Effect of added helium on phenol conversion at $442^{\circ} \mathrm{C}$ and 207 bar.

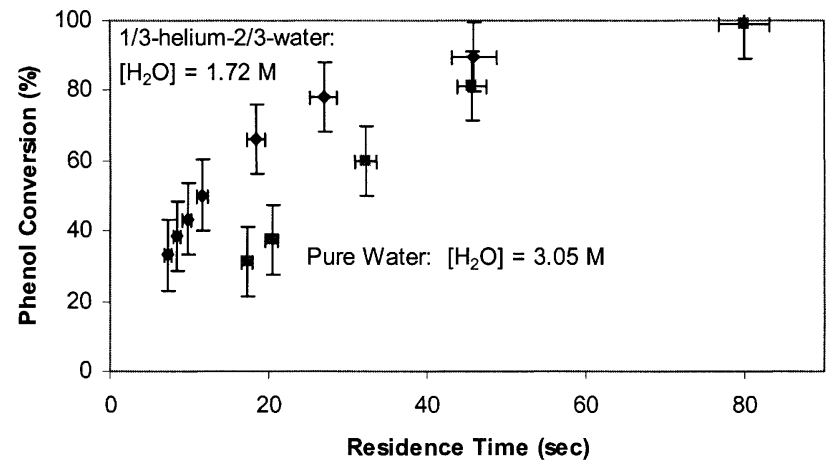

Figure 3. Effect of added helium on phenol conversion at $420^{\circ} \mathrm{C}$ and 141 bar.

objective, in this article, to elucidate the different physical or chemical roles that water molecules might be playing in this reaction. This subject is the focus of ongoing research, and the review by Akiya and Savage (2002) identifies many different potential roles for water.

\section{Effect of added helium}

We conducted phenol SCWO experiments with and without added helium at nominally identical temperatures, pressures, and initial concentrations of phenol and oxygen. These experiments explored different residence times for each set of reaction conditions, so complete phenol conversion profiles were obtained. Figures $2-4$ provide representative experimental results. Figure 2 shows that the phenol conversion at $442^{\circ} \mathrm{C}$ and 207 bar was about $44 \%$ in pure SCW at a residence time of $28 \mathrm{~s}$. The conversion in a $1 / 3$-helium-2/3-water mixture at the same conditions and residence time was about $90 \%$. All of the data in these figures, and throughout the entire data set, consistently show that the phenol conversion in the helium-water mixture exceeded the conversion obtained in pure SCW at the same residence time.

The rate-enhancing effect was also observed with regard to the ultimate oxidation products, $\mathrm{CO}$ and $\mathrm{CO}_{2}$. Figure 5 demonstrates that the introduction of helium increases the phenol conversion and the $\mathrm{CO}+\mathrm{CO}_{2}$ yield by approximately

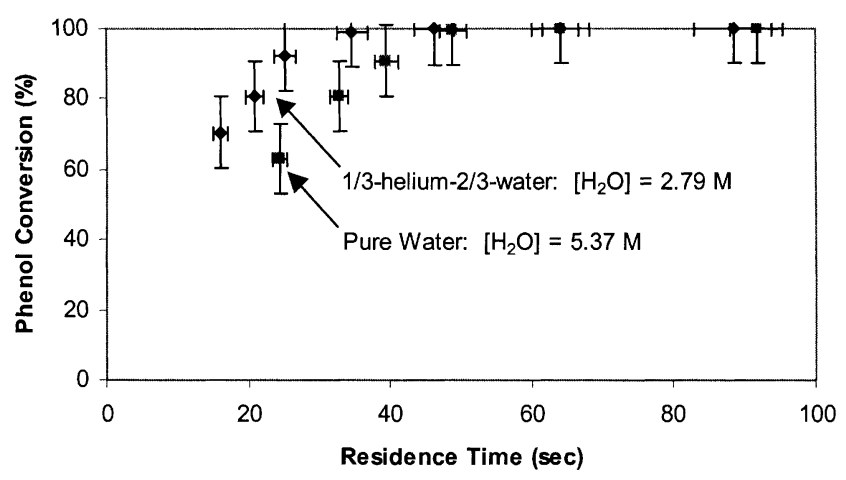

Figure 4. Effect of added helium on phenol conversion at $465^{\circ} \mathrm{C}$ and 241 bar. 


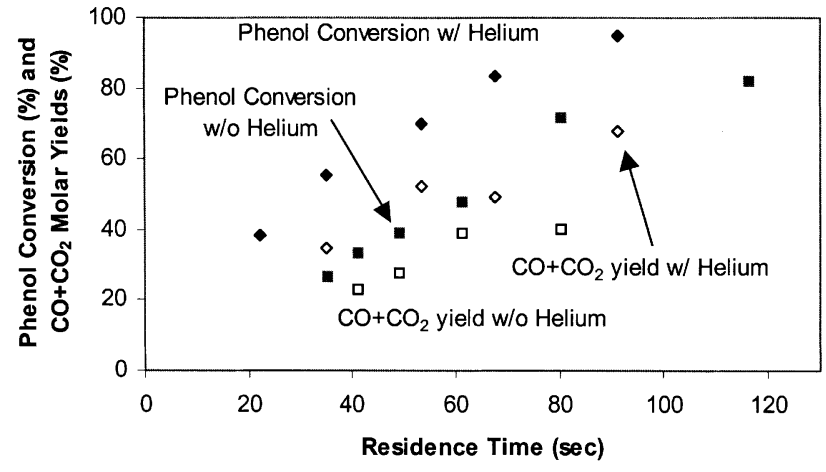

Figure 5. Effect of added helium on $\mathrm{CO}+\mathrm{CO}_{2}$ yields at $420^{\circ} \mathrm{C}$ and 241 bar.

the same amount. The experimental results consistently show that, other factors being equal, adding helium to the reaction medium increases the conversion of phenol and the yield of carbon oxides under the SCWO conditions tested.

We were surprised by the dramatic effect of the helium addition on the phenol conversion. Moreover, the higher conversion in the helium-water medium was the opposite of the result we expected, because previous experimental work in pure SCW performed in our laboratory consistently showed that decreasing the water density (by reducing the system pressure) decreased the phenol conversion. Therefore, we did some additional work to convince ourselves (and skeptical readers) that the experimental results we report herein are reliable.

We first compared experimental results reported by previous investigators in our research group to those obtained in this work. Figures 6 and 7 compare the experimental results of this work with those previously reported by Gopalan and Savage (1995) and Li et al. (1992) at approximately the same reaction conditions. The data at longer residence times are from the present investigation and those at the shorter residence times are from the literature. The results are similar, and the recent data appear to be a continuation of the same curves that appear in the earlier data.

Error Analysis. Having satisfied ourselves that the new data in pure SCW were consistent with those from previous studies, we next explored other factors. One possibility is that the differences in phenol conversion we report for the

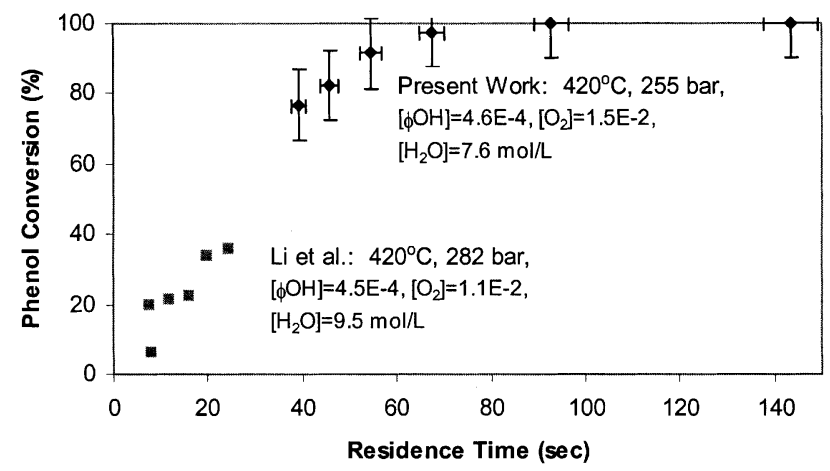

Figure 6. Comparison of literature data (Li et al., 1992) to present work.

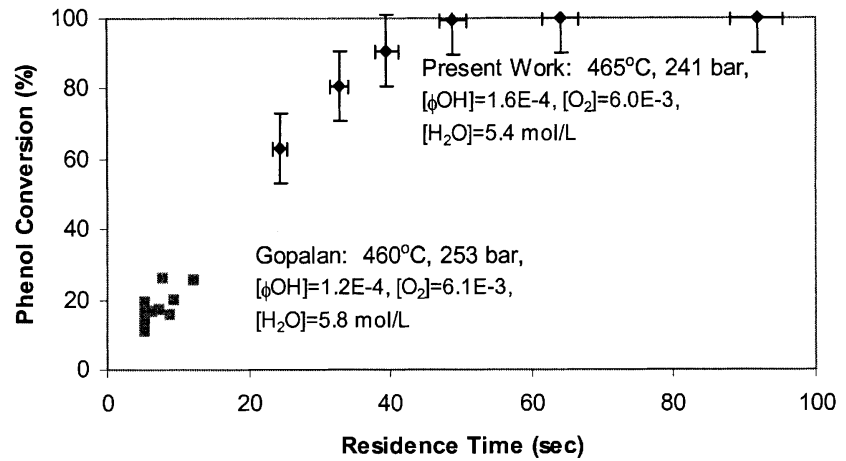

Figure 7. Comparison of literature data (Gopalan and Savage, 1995) to present work.

runs with and without helium can be attributed to experimental uncertainty.

We performed two separate error analyses to determine whether the differences observed with the addition of helium to the reaction medium were statistically significant. One approach was to determine the $95 \%$ confidence interval from five experiments performed at the same nominal reaction conditions $\left(442^{\circ} \mathrm{C}, 241\right.$ bar, $[\phi \mathrm{OH}]_{o}=0.19 \pm 0.01 \mathrm{mmol} / \mathrm{L}$, $\left.\left[\mathrm{O}_{2}\right]_{O}=6.6 \pm 1.6 \mathrm{mmol} / \mathrm{L}\right)$. The absolute uncertainty, at the $95 \%$ confidence level, for the phenol conversion at 36 and 43 s was $\pm 10.5 \%$ and $\pm 9.7 \%$ respectively. These confidence intervals represent a worst-case scenario because some of the "uncertainty" is due to the $25 \%$ variation in the oxygen concentration. Nevertheless, all of the figures in this article include error bars with an absolute uncertainty in conversion of $10 \%$.

The second approach was to determine the uncertainties associated with the different experimental measurements and then propagate those uncertainties through the equations used to calculate both the phenol conversion and the residence time. This approach led to a linear relationship between the phenol conversion (X) and its absolute error (Abs Error $=0.0834(1-X))$. At the $95 \%$ confidence level, the absolute uncertainty at a conversion of $20 \%$ is $\pm 6.7 \%$. The absolute uncertainty at a conversion of $90 \%$ is $\pm 0.9 \%$. We believe that this equation is the best representation of the actual errors incurred during data collection. Again, however, in the interest of being conservative, we used a value of $10 \%$ on all figures.

We also performed a propagation of errors analysis for the reactor residence time. We first considered systematic error due to uncertainty in the tubing radius. The manufacturer specified the uncertainty in the radius to be $\pm 10 \%$. This uncertainty leads to a $\pm 20 \%$ relative uncertainty in the residence time. It is important to keep in mind that this $20 \%$ uncertainty is a systematic and not a random uncertainty. That is, all of the results would be shifted by the same relative amount of time if the true reactor volume differed from the value we used to calculate the residence time. This systematic uncertainty in the residence time should only be taken into account when comparing the specific results herein with published results for phenol SCWO from different reactors. We also performed an uncertainty analysis to estimate the random error in the reactor residence time using the propa- 
Table 1. Reynolds and Peclet Numbers at $442^{\circ} \mathrm{C}$ and 241 Bar

\begin{tabular}{lcc}
\hline \multicolumn{1}{c}{ Reaction Medium } & Reynolds No. & Peclet No. \\
\hline Water and helium (lowest flow rate) & 718 & 921 \\
Water (lowest flow rate) & 698 & 1,342 \\
Water and helium (highest flow rate) & 4,582 & 3,750 \\
Water (highest flow rate) & 4,470 & 3,437 \\
\hline
\end{tabular}

gation-of-errors technique. The relative uncertainty in the reactor residence time was $\pm 4.0 \%$ for reactions in pure water and $\pm 6.2 \%$ for reactions in the helium-water medium. The differences in the phenol conversions in Figures 2-4 obtained with and without helium clearly exceed the estimated uncertainty in the conversion. Therefore, we conclude that the differences apparent for the experiments with and without helium are real and not simply manifestations of experimental uncertainty.

Flow Regime. Another possible explanation for the results in Figures 2-4 is that the experiments in pure water were done in one flow regime (such as laminar flow), whereas the experiments in the helium-water mixture were performed in a different flow regime (such as plug flow).

Laminar flow behavior would serve to reduce the phenol conversion by promoting Aris-Taylor dispersion. Ramayya and Antal (1989) showed that the misuse of the plug-flow idealization for a laminar flow reactor could erroneously increase the calculated rate constant $(k)$ by as much as $30 \%$. Plug flow is typically associated with Reynolds numbers greater than 4,000, indicating turbulent flow, whereas laminar flow is associated with Reynolds numbers below 2100. Table 1 lists the Reynolds numbers for the highest and lowest flow rates used in the present experiments. We estimated the diffusivity, density, and viscosity of helium-water mixtures (Poling, 2001), and used tabulated properties for pure SCW (Haar et al., 1984). Table 1 shows that the Reynolds numbers for the two different reaction media are comparable for both the low and high flow rates. This correspondence of the Reynolds numbers means that even if the flow is laminar at long residence times, it is laminar for both pure water and the helium-water mixture.

Moreover, a more thorough analysis revealed that the plug-flow idealization is reasonable even at the lowest flow rates. The criteria developed by Cutler et al. (1988) were used in this effort to assess the validity of the plug-flow approximation. Of the nine criteria presented, all were met in each of our experiments except for criterion number 5, which deals specifically with radial diffusion and surface reactions. Cutler et al. (1988) noted that this criterion need not be met as long as criterion 7 is met, and surface reactions are insignificant.

The Peclet number is another dimensionless number that can be used to assess the validity of the plug-flow approximation (as opposed to dispersed plug flow). The Peclet number is a measure of the relative rates of convective and dispersive fluid transport. The Peclet numbers in Table 1 are sufficiently large to allow the conclusion that convection is much more prevalent than dispersion (Fogler, 2001). Hence, the Peclet number analysis lends additional evidence that flow through the experimental tubular reactor can be reasonably approximated as plug flow.
Table 2. Mixing Tee Reynolds Numbers at $442^{\circ} \mathrm{C}$ and 241 Bar

\begin{tabular}{lc}
\hline \multicolumn{1}{c}{ Preheater Solvent } & Reynolds No. \\
\hline Water and helium (lowest flow rate) & 730 \\
Water (lowest flow rate) & 677 \\
Water and helium (highest flow rate) & 4,869 \\
Water (highest flow rate) & 4,513 \\
\hline
\end{tabular}

Based on this inspection of the Reynolds numbers, published criteria for plug flow, and Peclet numbers, we conclude that the experimental tubular flow reactor can be reasonably approximated as a plug-flow reactor, regardless of whether the reactor medium is water or a helium-water mixture.

Mixing Effects. One final factor we considered that might contribute to the differences observed in Figures 2-4 is differences in mixing efficiency for experiments with and without added helium. There were two different mixing tees in the reactor/preheater, and it is conceivable that the presence or absence of helium might affect the rate at which the oxidant and organic streams mixed to form a homogeneous fluid phase. The Reynolds number is the most important parameter related to the time required for complete mixing (Phenix et al., 2002). Table 2 shows the range of Reynolds numbers that corresponded to flow through the mixing tees in our experiments. It is clear that the Reynolds numbers for the two different reaction media were very similar, suggesting that the mixing efficiency should be similar for the two media. We note, however, that the Reynolds numbers were always less than 10,000 , the value that Phenix et al. identify as the minimum required to minimize mixing-induced induction times.

\section{Effect of temperature}

The experimental plan we employed also explored the effect of reactor temperature on the phenol conversion, both in pure water and in the helium-water mixture. Figures 8 and 9 provide representative experimental results. Both figures show that, for a given residence time, the phenol conversion increased as the temperature increased. This trend is expected because increasing temperature would increase the rate constant for the reaction.

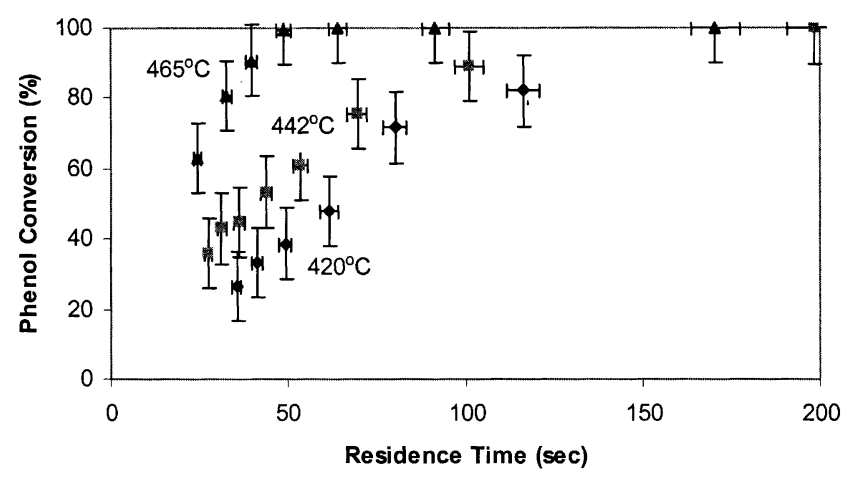

Figure 8. Effect of temperature on phenol conversion at 241 bar (pure SCW). 


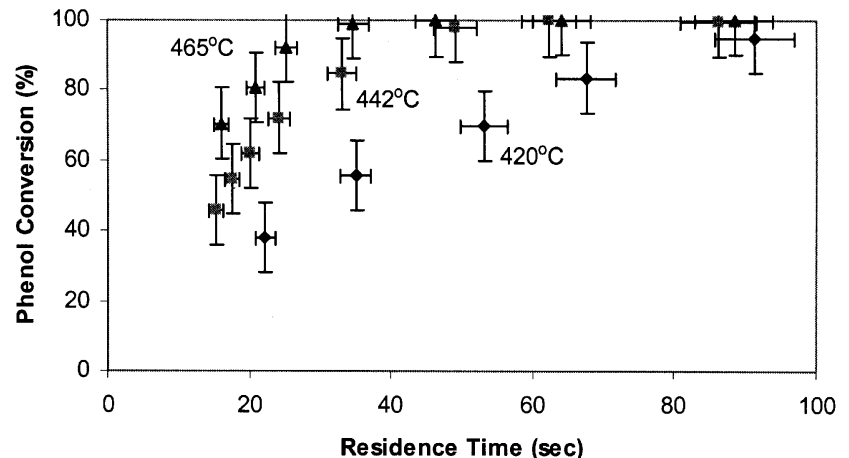

Figure 9. Effect of temperature on phenol conversion at 241 bar (helium-water medium).

Figures 8 and 9 also allow one to compare the relative effects of added helium and increased temperature on the phenol conversion. Figure 8 shows that increasing the temperature from $442^{\circ} \mathrm{C}$ to $465^{\circ} \mathrm{C}$ increases the phenol conversion at $50 \mathrm{~s}$ from about $50 \%$ to nearly $100 \%$. Figure 9 shows that this same increase in conversion was achieved simply by adding helium to the reaction medium at $442^{\circ} \mathrm{C}$. We point out this effect to demonstrate the magnitude of the change in the phenol conversion that occurred when helium was added to the reaction medium.

\section{Effect of feed concentrations}

We conducted a set of experiments at $442^{\circ} \mathrm{C}$ and 207 bar wherein the phenol concentration in the feed stream was varied until it was about $70 \%$ higher than its base-case concentration. The oxidant concentration was also increased to maintain a roughly $400 \%$ stoichiometric excess. Thus, both the phenol and oxygen concentrations increased, but the stoichiometric ratio of oxygen to phenol remained roughly unchanged.

Figure 10 shows that increasing the phenol and oxygen concentrations increased the phenol conversion. For example, in pure SCW at a 54-s residence time, the conversion was about $70 \%$ at the lower concentration and nearly $100 \%$ at the higher concentration. Likewise, in a helium-water mixture, the higher reactant concentrations in the feed stream

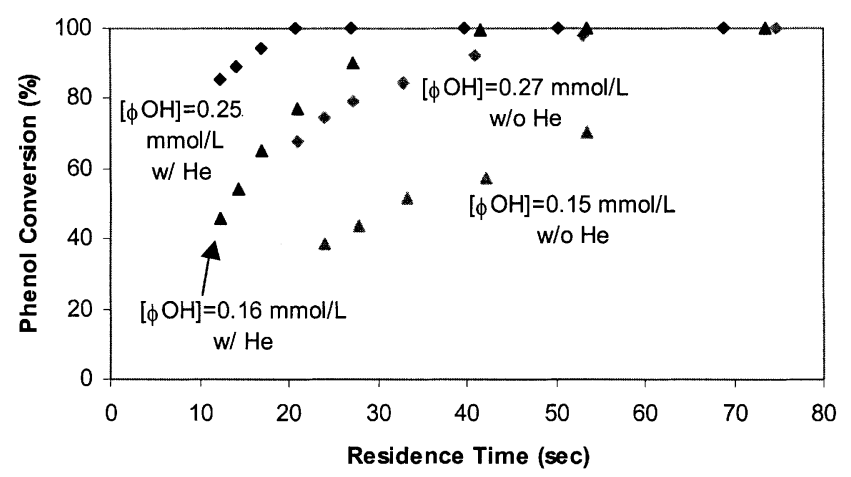

Figure 10. Effect of feed concentrations on phenol conversion at $442^{\circ} \mathrm{C}$ and 207 bar.

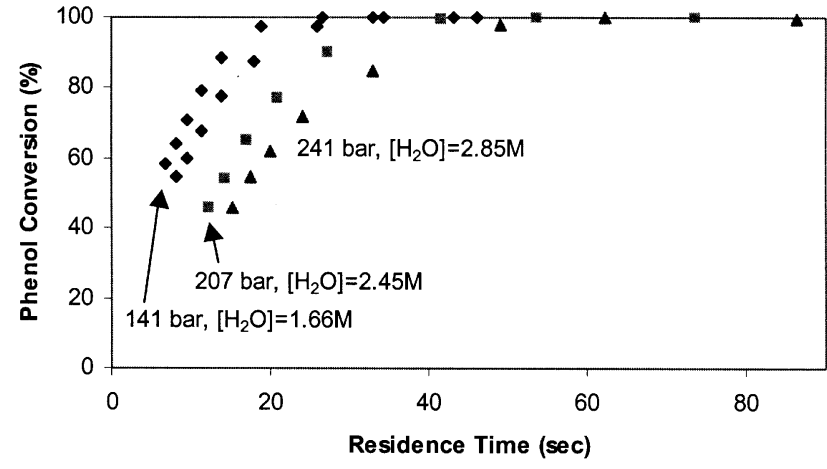

Figure 11. Phenol conversion in a helium-water medium at $442^{\circ} \mathrm{C}$ and different pressures/water concentrations.

resulted in a higher phenol conversion. This behavior is fully consistent with previous kinetic studies of phenol oxidation in supercritical water (Gopalan and Savage, 1995; Koo et al., 1997; Thornton and Savage, 1992; Oshima et al., 1998; Krajnc and Levec, 1996). Figure 10 demonstrates why it is imperative to compare experimental results obtained at the same phenol and oxygen concentrations.

\section{Effects of pressure}

The experimental plan allowed us to identify the effect of the system pressure on the phenol conversion, both in pure water and in the helium-water mixture. Figures 11 and 12 provide representative experimental results, in this case from experiments at $442^{\circ}$ and $420^{\circ} \mathrm{C}$, respectively. Figure 11 shows that, for a given residence time, the phenol conversion obtained in a helium-water mixture increased as the pressure and water density decreased. The same trend is apparent in Figure 12 when comparing 207 to 241 bar, which provides data from experiments in pure SCW. Other investigators have also observed an influence of the system pressure on phenol SCWO rates. This influence has typically been correlated by including the water concentration in the rate equation. The implicit assumption was that the water concentration, and not the pressure, was the more important variable, though both changed together. The next subsection explores water concentration effects in more detail.

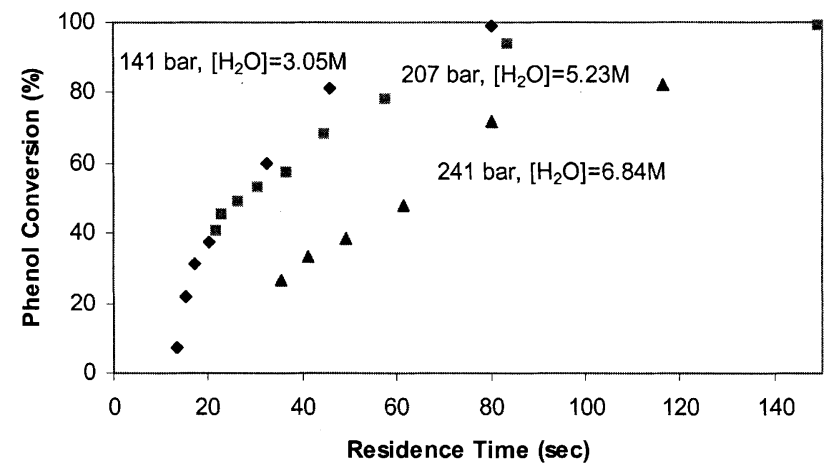

Figure 12. Phenol conversion in pure $\mathrm{SCW}$ at $420^{\circ} \mathrm{C}$ and different pressures/water concentrations. 


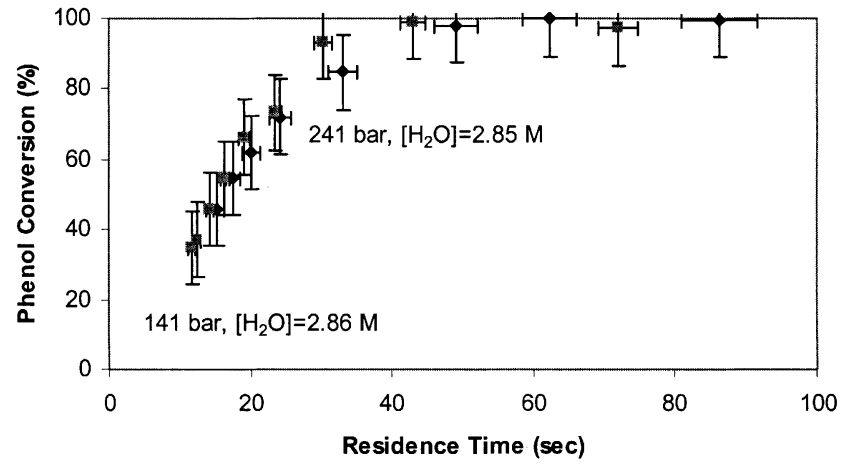

Figure 13. Phenol conversions at similar water concentrations but different pressures $\left(442^{\circ} \mathrm{C}\right)$.

\section{Effect of water concentration}

The data in Figures 11 and 12 were from experiments in which the system pressure was varied. Because the reaction medium is a compressible fluid, changing the system pressure also changes the concentration of the components. The concentrations of phenol and oxygen were roughly the same in all of the experiments, but the concentration of water changed as the system pressure changed. The experiments done with added helium provide data that allow us to decouple the effects of pressure and water concentration on phenol conversion.

Figures 13 and 14 display the phenol conversions observed from experiments at different system pressures, but with roughly the same water concentrations by combining a purewater experiment with a helium-water experiment. These figures show that at a given water concentration the phenol conversion is relatively insensitive to the system pressure. Therefore, it appears that it is the water concentration and not the system pressure that is responsible for the trends in Figures 11 and 12. Koo et al. (1997) reached the same conclusion in their experiments with added helium. Figures 13 and 14 also demonstrate that the addition of $33 \%$ helium to the solvent had no statistically significant effect on the disappearance of phenol. This observation suggests that the introduction of helium into the reaction medium affects the reaction primarily by changing the water concentration, and not by altering the physical properties of the medium.

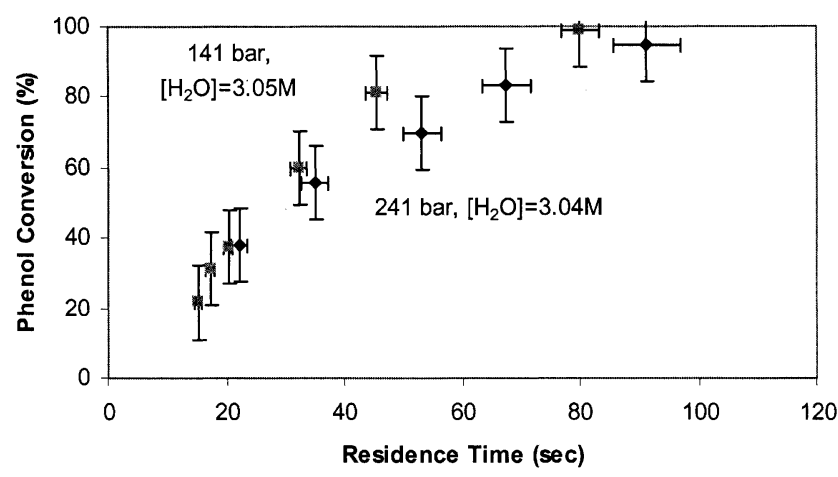

Figure 14. Phenol conversions at similar water concentrations but different pressures $\left(420^{\circ} \mathrm{C}\right)$.

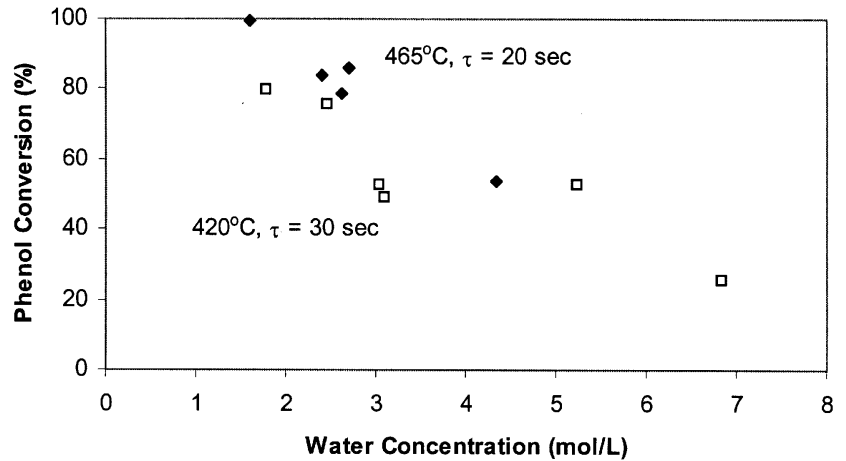

Figure 15. Effect of water concentration on phenol conversion.

To verify that the water concentration (and not pressure) has the larger influence on the phenol conversion, we offer Figure 15. This figure displays the phenol conversion at a given temperature and reactor residence time as a function of the water concentration. When necessary, we estimated the phenol conversion at a particular residence time via linear interpolation of the experimental data. Half of the data at a given temperature and residence time are from experiments in pure SCW (at different pressures), and the other half are from experiments in a helium-water mixture (at different pressures). The data at both $\tau=20$ and $30 \mathrm{~s}$ show that as the water concentration increases, the phenol conversion decreases. It is clear that the water concentration plays an important role in phenol SCWO kinetics at these reaction conditions.

There have been four previous articles that report the effect of water concentration on phenol conversion at supercritical conditions (Gopalan and Savage, 1995; Koo et al., 1997; Thornton and Savage, 1992; Oshima et al., 1998). Of the previous reports, only Oshima et al. (1998) obtained results consistent with ours regarding the inhibiting influence of the water concentration on the phenol disappearance rate. Thus, there continues to be a lack of agreement in the field regarding the qualitative effect of water concentration on phenol disappearance during SCWO. Moreover, there does not appear to be any consistent difference in the experimental conditions used in the different studies that might account for the disparate results. We note in this regard that the present work employed the lowest pressures and water densities, as well as the largest reactor.

\section{Conclusions}

Phenol disappearance during SCWO depends upon the water concentration, but not the system pressure. Under the conditions investigated herein, decreasing the water concentration at constant pressure increased the phenol conversion. A change in the water concentration from $5.0 \mathrm{M}$ to $2.5 \mathrm{M}$ increased the phenol conversion as much as, and sometimes more than, a $45^{\circ} \mathrm{C}$ temperature increase. Determining the exact role(s) of water under various SCWO conditions is the focus of ongoing research as we examine possible explanations such as solvation effects, catalysis, and the role of water as a collision partner and reactant/product. Although water concentration is not widely perceived to be an important vari- 
able in SCWO processes, it could be key to the optimization of SCWO reactions. Controlling the water concentration in SCWO processes might provide a way to enhance and control SCWO reaction rates.

\section{Acknowledgments}

We acknowledge financial support for this work from the National Science Foundation (CTS-9903373). We also thank Professor Mark Stadtherr at Notre Dame for providing calculated critical properties for helium-water mixtures.

\section{Literature Cited}

Akiya, N., and P. E. Savage, "Roles of Water for Chemical Reactions in High-Temperature Water," Chem. Rev., 102, 2725 (2002).

Chang, K. C., L. X. Li, and E. F. Gloyna, "Supercritical Water Oxidation of Acetic Acid by Potassium Permanganate," J. Hazard Mater., 33, 51 (1993).

Cutler, A. H., M. J. Antal, Jr., and M. Jones, Jr., “A Critical Evaluation of Tubular-Flow Reactor Data," Ind. Eng. Chem. Res., 27, 691 (1988).

Dell'Orco, P., B. Foy, E. Wilmanns, L. Le, J. Ely, K. Patterson, and S. Buelow, "Hydrothermal Oxidation of Organic Compounds by Nitrate and Nitrite," Innovations in Supercritical Fluids: Science and Technology, K. W. Hutcheson and N. R. Foster, eds., ACS Symp. Ser. 608, 179 (1995).

Fogler, H. S, Elements of Chemical Reaction Engineering, 3rd ed., Prentice Hall, Upper Saddle River, NJ, p. 877 (2001).

Gopalan, S., and P. E. Savage, "A Reaction Network Model for Phenol Oxidation in Supercritical Water," AIChE J., 41, 1864 (1995).

Haar, L., J. S. Gallagher, and G. Kell, NBS/NRC Steam Tables: Thermodynamic and Transport Properties and Computer Programs for Vapor and Liquid States of Water in SI Units, Hemisphere, New York (1984).

Holgate, H. R., and J. W. Tester, "Oxidation of Hydrogen and Carbon Monoxide in Sub- and Supercritical Water: Reaction Kinetics, Pathways, and Water-Density Effects: 1. Experimental Results," $J$. Phys. Chem., 98, 800 (1994).

Koo, M., W. K. Lee, and C. H. Lee, "New Reactor System for Supercritical Water Oxidation and Its Application on Phenol Destruction," Chem. Eng. Sci., 52, 1201 (1997).

Krajnc, M., and J. Levec, "On the Kinetics of Phenol Oxidation in Supercritical Water," AIChE J., 42, 1977 (1996).

Krajnc, M., and J. Levec, "The Role of Catalyst in Supercritical Water Oxidation of Acetic Acid," Appl. Catal. B. Environ., 13, 93 (1997).

Lee, D. S., and E. F. Gloyna, "Hydrolysis and Oxidation of Acetamide in Supercritical Water," Environ. Sci. Technol., 26, 1587 (1992).

Lee, D. S., K. S. Park, Y. W. Nam, Y. C. Kin, and C. H. Lee, "Hydrothermal Decomposition and Oxidation of p-Nitroaniline in Supercritical Water," J. Hazard Mater., 56, 247 (1997).
Lee, D. S., and S. D. Park, "Decomposition of Nitrobenzene in Supercritical Water," J. Hazard Mater., 51, 67 (1996).

Li, R., T. D. Thornton, and P. E. Savage, "Kinetics of $\mathrm{CO}_{2}$ Formation from the Oxidation of Phenols in Supercritical Water," Environ. Sci. Technol., 26, 2388 (1992).

Li, R. K., P. E. Savage, and D. Szmukler, "2-Chlorophenol Oxidation in Supercritical Water: Global Kinetics and Reaction Products," AIChE J., 39, 178 (1993).

Marrone, P. A., T. A. Arias, W. A. Peters, and J. W. Tester, "Solvation Effects on Kinetics of Methylene Chloride Reactions in Suband Supercritical Water: Theory, Experiment, and Ab Initio Calculations," J. Phys. Chem. A., 102, 7013 (1998).

Martino, C. J., and P. E. Savage, "Supercritical Water Oxidation Kinetics, Products, and Pathways for $\mathrm{CH} 3-$ and $\mathrm{CHO}-S u b s t i t u t e d$ Phenols," Ind. Eng. Chem. Res., 36, 1391 (1997).

Martino, C. J., P. E. Savage, and K. Kasiborski, "Kinetics and Products from $o$-Cresol Oxidation in Supercritical Water," Ind. Eng. Chem. Res., 34, 1941 (1995).

Meyer, J. C., P. A. Marrone, and J. W. Tester, "Acetic Acid Oxidation and Hydrolysis in Supercritical Water," AIChE J., 41, 2108 (1995).

Oshima, Y., K. Hori, M. Toda, T. Chommanad, and S. Koda, "Phenol Oxidation Kinetics in Supercritical Water," J. Supercrit. Fluids, 13, 241 (1998).

Phenix, B. D., J. L. DiNaro, J. W. Tester, J. B. Howard, and K. A. Smith, "The Effects of Mixing and Oxidant Choice on Laboratory-Scale Measurements of Supercritical Water Oxidation Kinetics," Ind. Eng. Chem. Res., 41, 624 (2002).

Poling, B. E., The Properties of Gases \& Liquids, McGraw-Hill, New York (2001).

Ramayya, S. V., and M. J. Antal, Jr., "Evaluation of Systematic Error Incurred in the Plug Flow Idealization of Tubular Flow Reactor Data," Energy Fuels, 3, 105 (1989).

Rice, S. F., R. R. Steeper, and J. D. Aiken, "Water Density Effects on Homogeneous Water-Gas Shift Reaction Kinetics," J. Phys. Chem. A., 102, 2673 (1998).

Savage, P. E., and M. A. Smith, "Kinetics of Acetic Acid Oxidation in Supercritical Water," Environ. Sci. Technol., 29, 216 (1995).

Sretenskaja, N. G., R. J. Sadus, and E. U. Franck, "High-Pressure Phase Equilibria and Critical Curve of the Water + Helium System to $200 \mathrm{MPa}$ and $723 \mathrm{~K}$," J. Phys. Chem., 99, 4273 (1995).

Steeper, R. R., S. F. Rice, I. M. Kennedy, and J. D. Aiken, "Kinetics Measurements of Methane Oxidation in Supercritical Water," $J$. Phys. Chem., 100, 184 (1996).

Stradi, B. A., J. F. Brennecke, J. P. Kohn, and M. A. Stadtherr, "Reliable Computation of Mixture Critical Points," AIChE J., 47, 212 (2001).

Thornton, T. D., and P. E. Savage, "Kinetics of Phenol Oxidation in Supercritical Water," AIChE J., 38, 321 (1992).

Manuscript received Mar. 25, 2002, and revision received July 18, 2002. 